\title{
Demandas fisiológicas de la competición en pádel. Physiological demands of competition in paddle.
}

\author{
Moisés de Hoyo Lora \\ Borja Sañudo Corrales \\ Luís Carrasco Páez \\ Dpto. de Educación Física y Deporte \\ Grupo de Investigación "Educación Física, Salud y Deporte (HUM-507)" \\ Universidad de Sevilla
}

Resumen

El objetivo del presente estudio es determinar las exigencias fisiológicas más relevantes de la práctica del pádel. Un total de 12 jugadores, varones de categoría nacional (edad, media \pm dt: $16.57 \pm$ 1.51 años) participaron de forma voluntaria en el estudio. Veinticuatro horas antes de competir, los sujetos realizaron una prueba máxima e incremental en tapiz rodante, determinándose diferentes parámetros cardiorrespiratorios, como el consumo de oxígeno (VO2) y la frecuencia cardiaca (FC). En la competición se obtuvieron los valores relativos al VO2 y FC. El VO2 alcanzó cifras inferiores al $50 \%$ del valor máximo alcanzado en la prueba sobre tapiz, mientras que la FC media representó, aproximadamente, un $74 \%$ de la FC máxima alcanzada en la prueba de laboratorio. Tras la definición de las características funcionales del pádel de competición, muy parecidas a las del tenis individual, se posibilita un mejor diseño de los programas de entrenamiento de los jugadores, recomendándose su práctica, además, a un amplio espectro de la población.

\section{abstract}

The aim of the present work is to determine the physiological exigencies of the practice of paddle. A total of 12 top level male players (age, mean \pm sd: $16.57 \pm 1,51$ years) participated in the study. Twenty-four hours before competition, the subjects carried out an incremental test on a treadmill, determining different cardiorespiratory parameters, as the oxygen uptake (VO2) and the heart rate (HR). In competition the VO2 and HR values were obtained. The VO2 reached values below $50 \%$ of the maximum that those reached in treadmill test, whereas the mean HR represented, approximately, $74 \%$ of the maximum HR reached in the same test. After the definition of the functional characteristics of paddle competition, very similar ones to those of individual tennis. A better design of the training programs of the players is made possible, recommending itself its practice, in addition, to a wide spectrum of the population.

Palabras clave: pádel, competición, consumo de oxígeno, frecuencia cardiaca.

Key words: paddle, competition, oxygen uptake and heart rate.

Correspondencia/correspondence: D. Moisés de Hoyo Lora

Facultad de Ciencias de la Educación. Avda. Ciudad Jardín 22. E-41005. Sevilla

E-mail: dehoyolora@us.es 


\section{Introducción}

$\mathrm{N}$

o hay duda del crecimiento popular del pádel como preferencia en la práctica de actividad física de personas de toda clase y condición. Además, el número de jugadores que participan en competiciones regladas ha experimentado un notable aumento en los últimos años, lo cual es una clara muestra del grado de aceptación en nuestra sociedad.

Este notable crecimiento de la práctica del pádel hace necesario un análisis pormenorizado de los elementos de los que consta, algo imprescindible para poder diseñar con mayor exactitud los programas de entrenamiento y mejorar el rendimiento de los jugadores. Centrándonos en los deportes de raqueta, numerosos autores han descrito la estructura de estas especialidades, así como las demandas fisiológicas que, en cada caso, se requieren para conseguir el éxito deportivo. En este sentido, destacan los trabajos realizados sobre tenis por Christmass et al. (1998), Ferrauti et al. (2001), Davey et al. (2003). En bádminton este tipo de investigaciones han sido desarrolladas por Cabello (2000), Torres et al. (2004) y Oswald (2006), mientras que Wells et al. (2004), Vuckovic et al. (2004) y Alvero et al. (2006) han definido la competición en squash teniendo en cuenta aspectos tanto fisiológicos como estructurales.

Sin embargo, hasta el momento, no se ha realizado ninguna investigación que permita una definición concreta de las características de la competición en pádel. De este modo, el objetivo del presente estudio es definir las exigencias fisiológicas así como las características estructurales más relevantes de su práctica, ofreciendo una información relevante de cara a la planificación del entrenamiento en este deporte.

\section{Material y Métodos}

Muestra

Un total de doce jugadores de categoría nacional, todos ellos varones y diestros (edad, media \pm dt: $16.57 \pm 1.51$ años; talla: $1.72 \pm 0.08 \mathrm{~m}$; masa corporal: $66.00 \pm 11.37 \mathrm{~kg}$; IMC o índice de masa corporal: $22.24 \pm 2.73 \mathrm{~kg} / \mathrm{m}^{2}$ ), participaron de forma voluntaria en el estudio. Su participación fue precedida de la lectura y firma de un consentimiento informado diseñado a tal efecto. El estudio además fue aprobado por el comité de ética de la Universidad de Sevilla. 


\section{Procedimiento}

\section{Determinación del consumo de oxígeno( $\left(V_{2 \max }\right)$ y otros parámetros cardiorrespiratorios.}

En las veinticuatro horas previas a la participación en un partido de competición, los jugadores participantes en el estudio fueron sometidos a una prueba máxima e incremental en tapiz rodante (Baum Electronic Ergo-run Medical 8) de cara a la determinación del $\mathrm{VO}_{2 m a ́ x}$ y de otras variables ergoespirométricas, como el umbral ventilatorio 2 (VT2) o umbral anaeróbico y la ventilación pulmonar. Además se calcularon los porcentajes del VT2 respecto al $\mathrm{VO}_{2 \text { máx }}$ (Imagen 1).

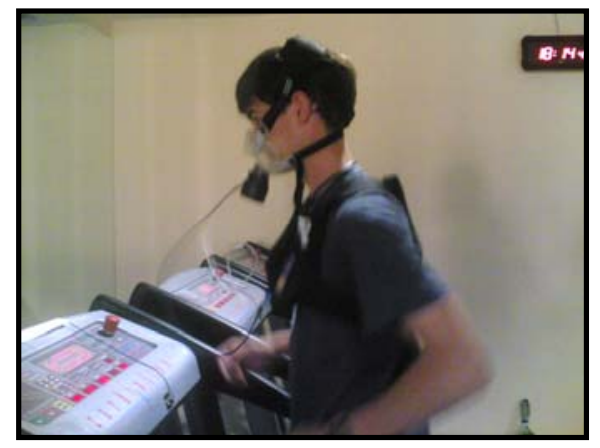

Antes de llevar a cabo esta prueba, los sujetos realizaron, a modo de calentamiento, carrera sobre el tapiz a una velocidad de $6 \mathrm{~km} / \mathrm{h}$, durante cinco minutos (inclinación: 1\%). Finalizado este periodo, la velocidad se situó en $8 \mathrm{~km} / \mathrm{h}$ para dar comienzo a la prueba, aumentando en $1 \mathrm{~km} / \mathrm{h}$ cada minuto hasta llegar al agotamiento o cumplir los criterios estándares de maximalidad (ACSM, 1999).

Imagen 1: Determinación de los parámetros fisiológicos en tapiz rodante.

La recogida de gases espirados durante la prueba de tapiz rodante y el juego real (Imagen 2) se efectuó con el analizador portátil MetaMax 3B (CORTEZ Biophysik GMBH, Germany), con tecnología "breath by breath". Los datos, recogidos a través de telemetría en un PC receptor, fueron analizados por el software Metasoft 3. Este sistema telemétrico permitió, además, obtener, con una frecuencia de 2-3 s, el registro de la frecuencia cardiaca (FC) durante toda esta prueba, pudiendo definir así la $\mathrm{FC}$ máxima $\left(\mathrm{FC}_{\text {máx }}\right)$ y establecer relaciones entre $\mathrm{FC}$ y varios de los parámetros respiratorios, como el $\mathrm{VO}_{2 \text { máx }} \mathrm{y}$ VT2.

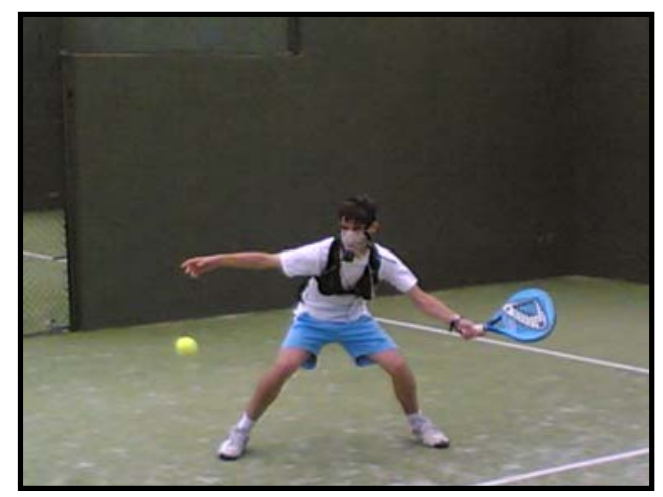

Imagen 2: Determinación de las demandas fisiológicas en juego real.

\section{Análisis estadístico}

Todas las variables fueron cuantificadas y registradas en las hojas de observación diseñadas a tal fin. La característica principal de estas hojas de observación que su simplicidad y practicidad, ya que a partir de ellas, los datos fueron transcritos y tratados estadísticamente con software SPSS 12.0. Este análisis estadístico se basó en medidas centrales y de dispersión, haciendo hincapié en la medida de frecuencias 


\section{Resultados}

Teniendo en cuenta las fases en las que hemos desarrollado nuestro estudio, se muestran a continuación los resultados más relevantes que se han obtenido.

En la Tabla 1 se muestran los parámetros fisiológicos resultantes de la prueba de laboratorio. Por su parte en la Tabla 2 podemos observar el análisis de los parámetros fisiológicos que pueden influir de manera más determinante en la práctica del pádel y que fueron obtenidos durante el juego.

Tabla 2. Análisis de los parámetros fisiológicos de los jugadores de pádel en laboratorio

\begin{tabular}{|r|c|c|c|c|c|c|}
\hline & $\mathrm{VO}_{2 \max }$ & $\mathrm{VT} 2(\mathrm{a})$ & $\mathrm{VT} 2(\mathrm{~b})$ & $\mathrm{FC}_{\max }$ & $\% \mathrm{VT} 2 / \mathrm{VO}_{2 \max }$ & $\mathrm{VE}$ \\
\cline { 2 - 7 } $\begin{array}{r}\text { Media } \\
\mathrm{SD}\end{array}$ & 55,64 & 184,14 & 46,57 & 200,43 & 83,53 & 74,33 \\
\cline { 2 - 7 } & 8,84 & 17,37 & 9,11 & 15,76 & 7,12 & 10,39 \\
\hline
\end{tabular}

$\mathrm{VO}_{2}=$ Consumo de oxígeno ( $\left.\mathrm{ml} / \mathrm{kg} / \mathrm{min}\right)$; $\mathrm{FC}=$ Frecuencia cardiaca; VT2 (a) en lat $/ \mathrm{min}$ y VT2 (b) en l/min; $\% \mathrm{VT} 2 / \mathrm{VO}_{\eta_{\mathrm{mav}}}=$ Porcentaie del consumo de oxígeno máximo donde se sitúa el umbral anaeróbico: VE=Ventilación (l/min)

Tabla 1. Análisis de los parámetros fisiológicos de los jugadores de pádel durante el juego

\begin{tabular}{|c|c|c|c|c|c|c|c|c|}
\hline \multirow{3}{*}{$\begin{array}{r}\text { Media } \\
\text { SD }\end{array}$} & $\mathrm{VO}_{2 \text { medio }}$ & $\% \mathrm{VO}_{2 \max }$ & $\mathrm{FC}_{\max }$ & $\mathrm{FC}_{\text {media }}$ & $\% \mathrm{FC} / \mathrm{FC}_{\max }($ Partido) & $\% \mathrm{FC}_{\max } \mathrm{Par} / \mathrm{Tapiz}$ & $\% \mathrm{VO}_{\text {2juego }} / \mathrm{VT} 2$ & METS \\
\hline & 24,06 & 43,73 & 169,72 & 148,30 & 87,38 & 84,90 & 52,52 & 153,05 \\
\hline & 6,95 & 11,04 & 18,41 & 13,63 & 5,10 & 9,16 & 15,50 & 38,63 \\
\hline
\end{tabular}

$\mathrm{VO}_{2}=$ Consumo de oxígeno; $\mathrm{FC}=$ Frecuencia cardiaca; $\% \mathrm{FC} / \mathrm{FC}_{\max }($ Partido)=\%FC media durante al juego respecto a la máxima del partido $\mathrm{FC}_{\max } \mathrm{Par} / \mathrm{Tapiz}=\mathrm{FC}$ máxima de partido respecto a la máxima del tapiz; \%VO2juego/VT2=Consumo de oxígeno medio respecto al umbral anaeróbico; METS=Tasa metabólica basal. (3,5 ml/kg/min)

Los resultados derivados del presente trabajo aportan una descripción inicial de las demandas fisiológicas que implica la práctica de este deporte.

La FC media durante el juego en pádel fue de $148.30 \pm 13.73$ lat $/ \mathrm{min}$, lo que supone un porcentaje del $73.99 \pm 4.65$ respecto a la $\mathrm{FC}_{\max }$ alcanzada en la prueba realizada en laboratorio. Por otro lado, la $\mathrm{FC}_{\max }$ registrada durante el partido (169.72 \pm 18.41 lat/min) supuso un porcentaje del $84.90 \pm 9.16$ respecto a la FC obtenida durante la prueba máxima de tapiz. Los datos que, de alguna manera, pueden contrastarse con los aquí obtenidos hacen referencia al tenis individual. En este sentido, Christmass et al., (1998) informan de una $\mathrm{FC}_{\max }$ de $189 \pm 3 \mathrm{lat} / \mathrm{min}$, alcanzando un $85.1 \pm 1.3 \%$ de la $\mathrm{FC}_{\max }$ alcanzada en laboratorio. En el estudio de Torres et al. (2004) la FC media desarrollada durante un partido de tenis individual fue de $158.4 \pm 8.5$ lat $/ \mathrm{min}$, alcanzando los $179.5 \pm 8$ lat/min en la práctica del bádminton. Por su parte, Alvero et al. (2006) registraron una FC media de $167 \pm 12$ lat/min en los ganadores y $175 \pm 9.56$ lat/min en los perdedores de varios partidos de squash. Es sorprendente que tanto la FC media como la $\mathrm{FC}_{\max }$ en bádminton, squash y tenis sean sensiblemente superiores a la $\mathrm{FC}_{\max }$ registrada en los jugadores de pádel participantes en este estudio. Atendiendo al $\mathrm{VO}_{2 \text { max }}$, König et al. (2001) registraron cifras en jugadores profesionales de tenis de 55 $\mathrm{ml} / \mathrm{kg} / \mathrm{min}$ en mujeres y de $65 \mathrm{ml} / \mathrm{kg} / \mathrm{min}$ en hombres. Por su parte, Christmass (1998), 
analizando a jóvenes tenistas (24 \pm 2 años) determinó un $\mathrm{VO}_{2 \text { máx }}$ de $53.4 \pm 1.8$ $\mathrm{ml} / \mathrm{kg} / \mathrm{min}$. Por su parte, Avron (1993) registró cifras entre 48 y $62 \mathrm{ml} / \mathrm{kg} / \mathrm{min}$ en jugadores de squash entre 27.2 y 30.6 años de edad. En cuanto al $\mathrm{VO}_{2}$ desarrollado durante la competición individual en tenis, Ferrauti et al. (2001), observaron unos valores de $25.6 \pm 2.8 \mathrm{ml} / \mathrm{kg} / \mathrm{min}$, cifras algo superiores al $50 \%$ del $\mathrm{VO}_{2 \text { máx. En el }}$ presente estudio se ha obtenido un $\mathrm{VO}_{\text {2máx }}$ de $55.6 \pm 8.83 \mathrm{ml} / \mathrm{kg} / \mathrm{min}$, cifras acordes a las anteriormente citadas independientemente de la especialidad deportiva practicada. Durante la competición de pádel, los jugadores desarrollaron un $\mathrm{VO}_{2}$ de $24.1 \pm 6.94$ $\mathrm{ml} / \mathrm{kg} / \mathrm{min}$, una cifra prácticamente similar a la observada en el tenis individual (Ferrauti, 2001).

\section{Conclusiones}

Hasta el momento, ningún estudio había analizado las características funcionales, un deporte cuya práctica ha aumentado espectacularmente durante los últimos años. De esta forma, y atendiendo a los objetivos propuestos, se ha determinado la exigencia fisiológica de su práctica.

La intensidad desarrollada durante la práctica del pádel se aproxima a la experimentada en la práctica del tenis individual. A pesar que el $\mathrm{VO}_{2}$ de los jugadores de pádel es similar al de los jugadores de tenis (tanto en valores máximos como relativos durante el juego) las exigencias reales de la práctica son diferentes. Esto es debido, probablemente, a una menor distancia recorrida por los jugadores de pádel en sus acciones, un aspecto que puede compensarse con un mayor número de acciones respecto al tenis individual.

Son necesarios nuevos estudios que acaben de definir completamente los requerimientos de la práctica del pádel, haciendo especial hincapié en los tipos de golpeos empleados, su frecuencia de aparición y la eficacia de los mismos. 


\section{Bibliografía}

Abraham, A. (1993). The physiological responses to squash play. Extraído el 25 de enero de 2007 desde http://www.sportsci.org/encyc/drafts/Squash.doc

Alvero, J.R., Barrera, J., Mesa, A. (2006). Correlations of physiological responses in squash players during competition. Proceedings of the IV World Congress of Science and Racket Sports. Alcoy: Alto Rendimiento.

American Collage of Sports Medicine - ACSM (1999). Manual ACSM para la valoración y prescripción del ejercicio. Barcelona: Paidotribo.

Cabello, D. (2000). Análisis de las características del juego en bádminton de competición. Su aplicación al entrenamiento. Tesis Doctoral. Granada: Universidad de Granada.

Christmass, M.A., Richmond S.E., Cable, N.T., Arthur, P.G., Hartmann, P.E. (1998). Exercise intensity and metabolic response in singles tennis. Journal of Sport Sciences, 16, 739-747.

Davey, P.R., Thorpe, R.D., Williams, C. (2003). Simulated tennis matchplay in a controlled environment. J ournal of Sport Sciences, 21, 459-467.

Ferrauti, A., Bergeron, M.F., Pluim, B.M., Weber, K. (2001). Physiological responses in tennis and running with similar oxygen uptake. European Journal of Applied Physiology, 85, 27-33.

König D, Hounker M, Schimd A, Halle M, Berg A, Kaul J. Cardiovascular metabolic and hormonal parameters in professional tennis players. Medicine and Science in Sports and Exercise 2001, 33(4): 654-658.

Oswald, E. (2006). A computer-aided comparison of the playing pattern of the world's top male players and Austrian top male players in single badminton. Proceedings of the IV World Congress of Science and Racket Sports. Alcoy: Alto Rendimiento.

Torres, G., Cabello, D., Carrasco, L. (2004). Functional differences between tennis and badminton in young sportsmen. A. Lees, J.F. Khan, I.W. Maynard (Eds.). Science and Racket Sports III (185-189). Oxon: Routledge.

Vuckovic, G., Dezman, B., Erculj, F., Kovacic, S., Pers, J. (2004). Differences between the winning and the losing players in a squash game in terms of distance covered. A. Lees, J.F. Khan, I.W. Maynard (Eds.). Science and Racket Sports III (202-207). Oxon: Routledge.

Wells, J., Robertson, C., Hughes, M., Howe, D. (2004). Performance profiles of elite men squash doubles match play. A. Lees, J.F. Khan, I.W. Maynard (Eds.). Science and Racket Sports III (196-201). Oxon: Routledge. 